\title{
Síndrome de encefalopatía posterior reversible. Presentación radiológica atípica
}

Posterior Reversible Encephalopathy Syndrome. Atypical radiological presentation

Síndrome de encefalopatia posterior reversível (PRES), apresentação radiológica atipica

Miguel Ángel Galván Gastélum, ${ }^{*}$ Jesús Cleofas Ramírez Campaña, ${ }^{*}$ Cibeles Arrambí Díaz, ${ }^{\ddagger}$ Jesús Ariel Morales Barraza*

\section{RESUMEN}

Introducción: El síndrome de encefalopatía posterior reversible (PRES por las siglas en inglés Posterior Reversible Encephalopathy Syndrome) es una entidad clínica caracterizada por cefalea, alteraciones visuales y crisis convulsivas. Ésta es una entidad subdiagnosticada, que tiene como fisiopatología la alteración del vaso de regulación cerebral y se puede presentar en féminas gestantes, lo que puede llegar a confundirse con eclampsia.

Caso clínico: Paciente de 20 años sin antecedentes patológicos, con embarazo de término normoevolutivo. Presentó ruptura prematura de membranas e ingresó para conducción de trabajo; se preparó a la paciente para analgesia obstétrica; sin embargo, previamente al procedimiento presentó crisis convulsivas de características tonicoclónicas generalizadas. Se realizó un manejo avanzado de la vía aérea y se realizó una cesárea de urgencia; se obtuvo producto único vivo y fue ingresada a la Unidad de Cuidados Intensivos. El perfil toxémico no reportó alteraciones y la tomografía computarizada inicial no mostró alteraciones estructurales. Se le retiró a la paciente la ventilación mecánica 12 horas después. Se realizó una resonancia magnética que mostró en fase T2, hiperintensidad subcortical parieto-occipital derecho. Permaneció en vigilancia y fue egresada a piso sin complicaciones ni secuelas neurológicas.

Conclusiones: El tratamiento del PRES va enfocado al control de la presión arterial, y el soporte orgánico y metabólico. No todos los enfermos con PRES se recuperan neurológicamente ad integrum; sin embargo, la proporción de pacientes que cursan sin secuelas es mayor en pacientes obstétricas. La resonancia magnética es el estudio de elección; el hallazgo característico es el edema simétrico de la materia blanca en la región occipital de los hemisferios cerebrales, en particular las regiones parieto-occipitales, no obstante, existen otro tipo de presentaciones radiológicas.

Palabras clave: Embarazo, encefalopatía, eclampsia, síndrome de encefalopatía posterior reversible (PRES), monitoreo por IRM.

\section{ABSTRACT}

Introduction: Posterior Reversible Encephalopathy Syndrome (PRES) is a clinical entity characterized by headache, visual disturbances and convulsive seizures. It is a sub-diagnosed entity that, due to its trigger being cerebral vasoregulation disorders, may present in pregnant women, which may be confused with eclampsia.

Clinical case: Patient of 20 years of age, without pathological antecedents, with term pregnancy, evolutionary norm. Who enters by exit of mucous plug, followed by premature rupture of membranes, so it is admitted for conduction of labor, however presents convulsive crisis, generalized clonic tonic. Advanced airway management is decided, and an emergency caesarean section is performed, obtaining a single live product, and the patient is admitted to the ICU. His toxemic profile was reported without alterations, his initial CT scan without structural alterations, and mechanical ventilation was removed twelve hours later. MRI is performed where subcortical right parieto-occipital hyperintensity is observed, being kept under surveillance and graduated to floor without complications.

Conclusions: The treatment of PRES syndrome is focused on organic and metabolic supports and mainly on tension figures. Not all are reversible, however, the pregnant patient is more frequent than present evolution without sequelae. Magnetic resonance is the study of choice for this clinical entity, and the typical findings are symmetrical edema of white matter in the posterior cerebral hemispheres, particularly the parieto-occipital regions, but there are variations. Keywords: Pregnancy, encephalopathy, eclampsia, Posterior Reversible Encephalopathy Syndrome (PRES), MRI monitoring.

\footnotetext{
* Hospital de Especialidades No. 2 del CMN Noroeste «Lic. Luis Donaldo Colosio Murrieta» del Instituto Mexicano del Seguro Social. Ciudad Obregón, Sonora.

‡ Hospital General Regional No. 1 del Instituto Mexicano del Seguro Social. Ciudad Obregón, Sonora
}

Recepción: 13/09/2019. Aceptación: 07/01/2020.

www.medigraphic.com/medicinacritica

\section{RESUMO}

Introduçao: A síndrome da encefalopatia posterior reversível (PRES) é uma entidade clínica caracterizada por dor de cabeça, distúrbios visuais e convulsões. É uma entidade subdiagnosticada, cuja fisiopatologia é a alteração do vaso de regulação cerebral, pode ocorrer em mulheres grávidas, o que pode ser confundido com eclampsia.

Caso clínico: Paciente de 20 anos, sem antecedentes patológicos, com gestação á término e evolução normal. Apresentou ruptura prematura de membranas e ingressou para indução do trabalho de parto, preparou-se para analgesia obstétrica, porém antes do procedimento apresentou convulsões com características tônicas clônicas generalizadas. Foi realizado manejo avançado das vias aéreas e cesariana de emergência, obtendo-se um único produto vivo, e foi admitida na Unidade de Terapia Intensiva. O perfil toxémico não relatou alterações, a tomografia computadorizada inicial não mostrou alterações estruturais e a ventilação mecânica foi removida doze horas depois. Foi realizada ressonância magnética que mostrou na fase $T 2$, hiperintensidade subcortical parieto-occipital direita. A paciente permaneceu sob vigilância e recebeu alta sem complicações ou sequelas neurológicas.

Conclusões: O tratamento do PRES, está focado no controle da pressão arterial, suporte orgânico e metabólico. Nem todos os pacientes com PRES se recuperam neurologicamente ad integrum, no entanto, a proporção de pacientes que não apresentam sequelas é maior em pacientes obstétricos. $A$ ressonância magnética é o estudo de eleição; o achado característico é edema simétrico de substância branca na região occipital dos hemisférios cerebrais, particularmente regiões parietooccipitais, porém existem outros tipos de apresentações radiológicas.

Palavras-chave: Gestação, encefalopatia, eclampsia, síndrome de encefalopatia posterior reversível (PRES), monitoração por RM.

\section{INTRODUCCIÓN}

El síndrome de encefalopatía posterior reversible (PRES por las siglas en inglés Posterior Reversible Encephalopathy Syndrome) es una entidad clínicoradiológica que fue descrita por primera vez en 1996 por Hinchey y colaboradores. ${ }^{1}$ Se puede presentar en pacientes inmunocomprometidas, con trastornos hipertensivos, neoplasias y con tratamientos oncológicos. . $^{2,3}$ Es una entidad rara o tal vez subdiagnosticada, que habitualmente se interpreta como eclampsia.

Clínicamente, se presenta como un cuadro neurológico agudo con crisis convulsivas (74\% de los casos) tanto focales como generalizadas, e incluso estatus epiléptico, encefalopatía, síntomas visuales (visión borrosa, hemianopsia o ceguera cortical) y cefalea. Los déficits focales son raros, salvo en los que se deben a urgencia hipertensiva, hipertensión arterial, y si está presente, es moderada o normal en $20 \%$ de los casos. ${ }^{4}$

La resonancia magnética es el estándar de oro para realizar el diagnóstico; en aquélla se encuentran múltiples variables de presentación que, incluso, la suma de ellas supera al $100 \%$. Por un lado, se reporta que en $24 \%$ de las pacientes, se presenta sin lesión paren- 
quimatosa aguda, sin embargo, en esta presentación en fase FLAIR (fluid-attenuated inversion recovery) se llega a presentar un signo de punto hiperintenso en la lesión; por otro lado, $25 \%$ de las lesiones se encuentran al tomar corteza de los limites parietal y occipital; $28 \%$ debuta con imagen de edema vasogénico observado en FLAIR como lesiones hiperintensas en T2 y $42 \%$ muestra imágenes compatibles con imágenes hemorrágicas intraparenquimatosa lobulares y profundas; por último, otro $28 \%$ evidencia lesiones combinadas de tipo isquémica y hemorrágica, que se localizan de manera más sensible en FLAIR axial y tomografía. 5,6

A pesar de la entidad diferencial, el tratamiento va enfocado a los soportes orgánicos, metabólico y, principalmente, a las cifras tensionales. No todos son reversibles, pero al menos en pacientes embarazadas es más frecuente que éstas evolucionen sin secuelas.

\section{CASO CLÍNICO}

Se presenta el caso de una mujer de 20 años, residente y originaria de Cd. Obregón, Sonora, México, empleada, con secundaria terminada, en unión libre y de creencia católica. Niega tener enfermedades crónicas, alergias, y sin hospitalizaciones; también niega hemotransfusiones.

Sus antecedentes ginecoobstétricos fueron: G1, C1, PO A0. El inició de la vida sexual activa fue a los 17 años, sin método de planificación familiar y sin ingesta de anticonceptivos; sólo ha mantenido dos parejas se-

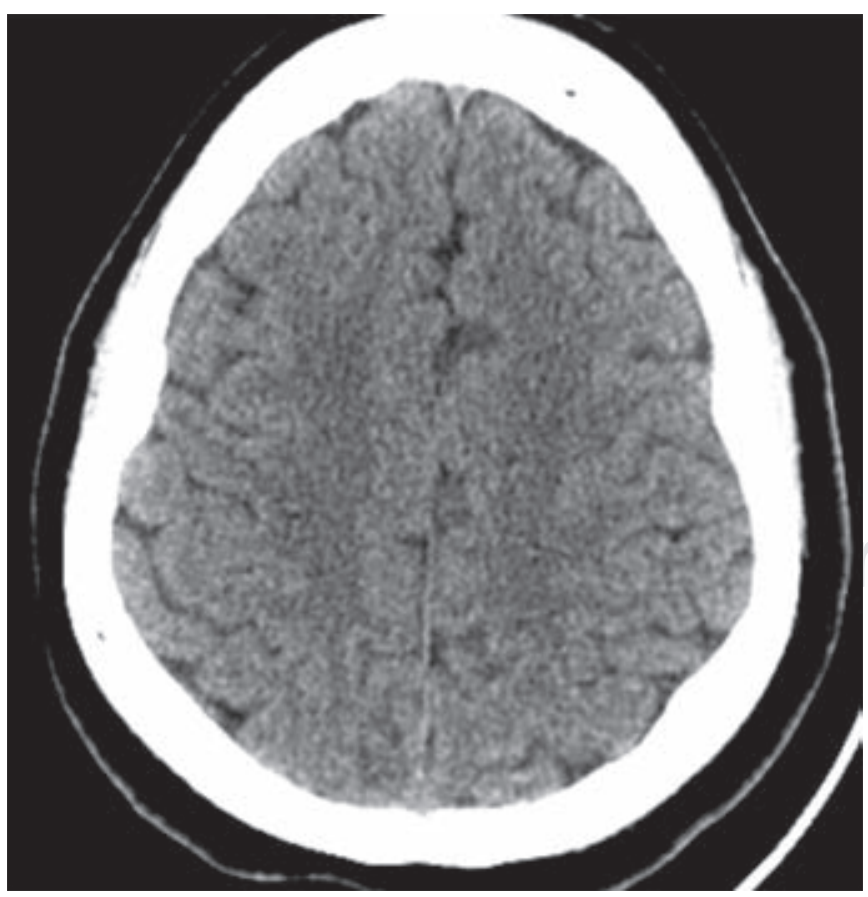

Figura 1: TC de cráneo fase simple, 24 horas de padecimiento actual. Se reporta con características normales.

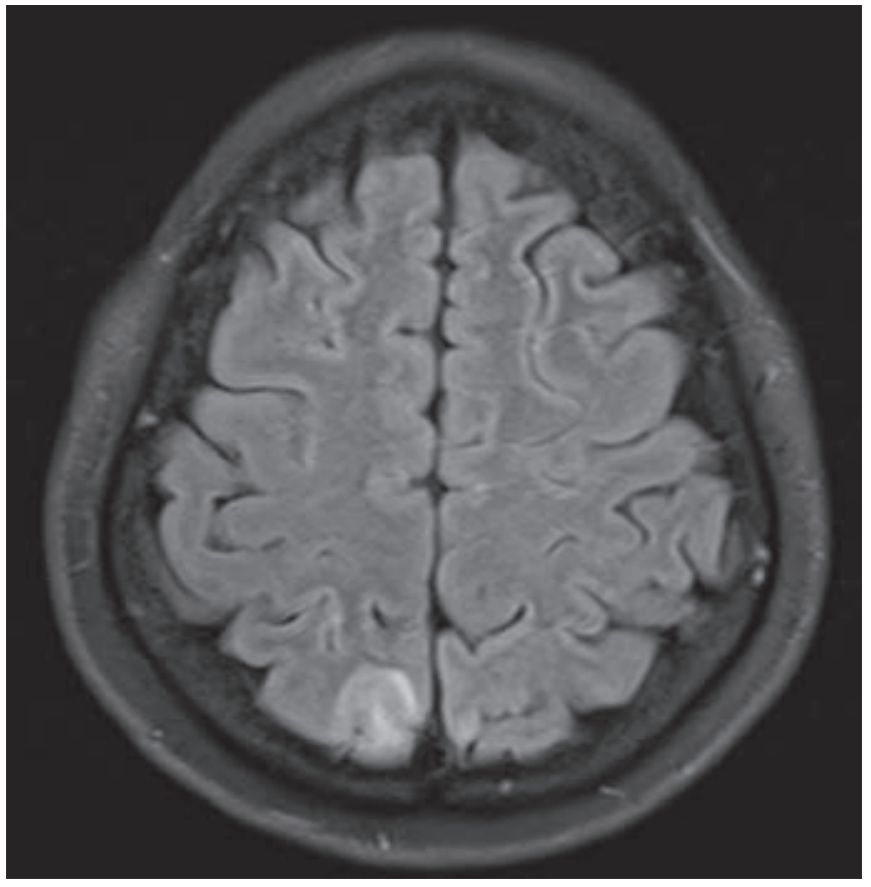

Figura 2: RM cráneo simple FLAIR fase T2. Zona hipertensa parieto-occipital derecho corte transverso a 48 horas de padecimiento actual.

xuales. Su embarazo fue normoevolutivo, de término, con ocho consultas gestacionales por el área de medicina familiar, dos por ginecología y obstetricia de control, y tres ultrasonidos obstétricos que fueron reportados sin alteraciones, además de exámenes paraclínicos de seguimiento gestacional normal.

Inició pródromos de trabajo de parto tres días previos a su ingreso al hospital; 24 horas previas al ingreso presentó salida de tapón mucoso que fue acompañado posteriormente con ruptura prematura de membranas, por lo que fue ingresada a tococirugía para conducción de trabajo de parto. Sus signos vitales fueron: T/A: 100/60 mmHg, FC: 80 , FR: $19, \mathrm{~T}: 37^{\circ} \mathrm{C}$ y SO${ }_{2}: 99 \%$.

Llegó a $8 \mathrm{~cm}$ de dilatación a las 24 horas de arrojar tapón mucoso y se le programó analgesia obstétrica, sin embargo, previamente a la realización del procedimiento, presentó crisis convulsivas tonicoclónicas generalizadas. La presión arterial posterior al evento fue la siguiente: T/A: 110/90 mmHg. Se le realizó intubación orotraqueal y cesárea de urgencia por sospecha de eclampsia y se obtuvo un producto único vivo; la prueba de Apgar fue 9/10, sin alteraciones.

Se ingresó a la paciente a la Unidad de Cuidados Intensivos. No tuvo alteraciones en el perfil toxémico. Se realizó tomografía computada del cráneo simple, la cual tuvo un reporte normal (Figura 1), y se retiró la sedación. A las 12 horas de presentar la crisis convulsiva fue liberada de la ventilación mecánica.

Al momento de la valoración neurológica no se encuentra plejia o paresias, sin alteraciones visuales, ce- 
falea, con coordinación íntegra. Se solicitó una resonancia magnética, la cual reportó en fase T2 zona de hiperintensidad parietal occipital derecho corte transverso y sagital (Figuras 2 y 3 ).

Se repitió a los ocho días la resonancia magnética de cráneo (Figura 4), la cual reportó características normales. Fue valorada por neurología y se confirmó diagnóstico de PRES. Se ajustó el tratamiento anticomicial con fenitoína y se egresó de la Unidad de Cuidados Intensivos sin mayor complicación ni secuelas neurológicas.

\section{DISCUSIÓN}

El síndrome de encefalopatía posterior reversible (PRES), previamente conocido como leucoencefalopatía posterior reversible, es un síndrome clínico radiográfico de origen variable que comparte síntomas similares a los de la eclampsia, por lo cual el diagnóstico diferencial es el de esta última patología, en caso de presentaciones atípicas de la eclampsia (sin proteinuria y sin hipertensión). En cuanto a su epidemiología, es un síndrome cada vez más reconocido en informes de casos y series de casos, sin embargo, la incidencia de PRES no se conoce. Por lo tanto, no existen guías de práctica clínica que normen su abordaje. ${ }^{1}$

La patogenia del síndrome de PRES no está clara, pero parece estar relacionada con la autorregulación cerebral desordenada y la disfunción endotelial. ${ }^{1}$ Debi-

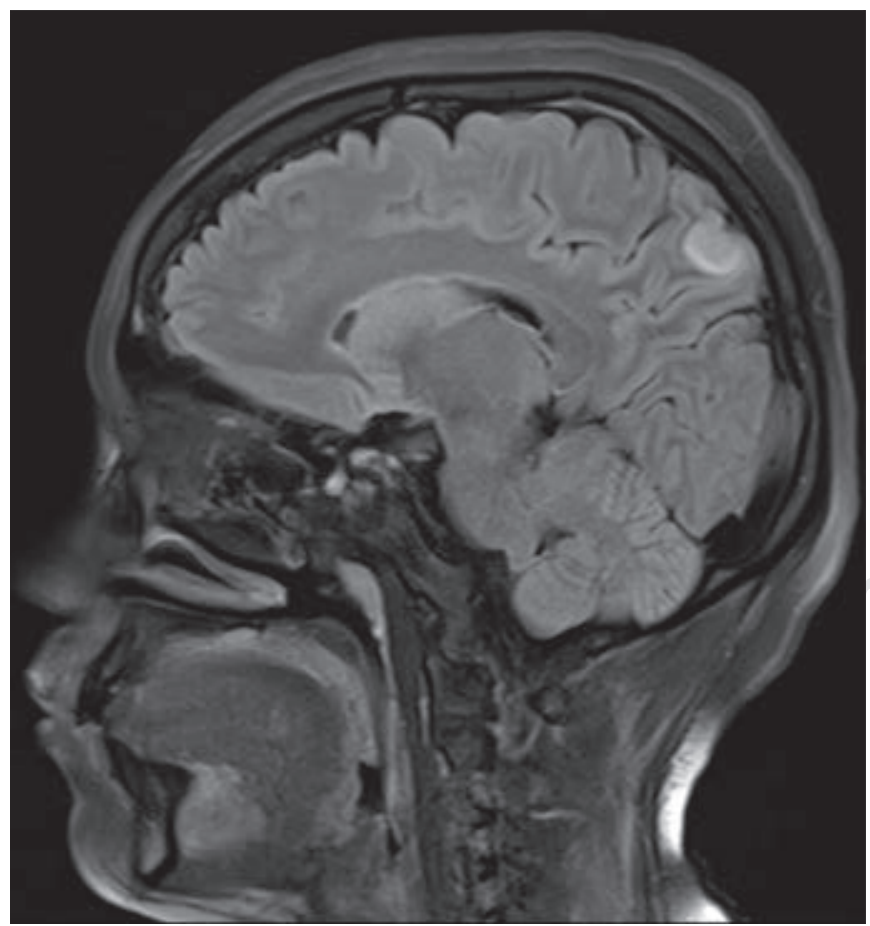

Figura 3: RM cráneo simple FLAIR fase T2, que muestra hiperintensidad parieto-occipital derecho corte sagital a 48 horas de padecimiento actual.



Figura 4: RM cráneo simple, FLAIR fase T2, ausencia de signos radiológicos de edema vasogénico. Control a ocho días de RM inicial.

do a la naturaleza heterogénea de este trastorno, puede ser que diferentes mecanismos sean etiológicamente importantes en diferentes situaciones clínicas. ${ }^{4,7}$

En cuanto a la fisiopatología, el tema aún es controvertido, sin embargo, se citan dos hipótesis: la teoría actual y más popular, sugiere que la hipertensión severa excede los límites de autorregulación, lo que lleva a un edema cerebral revolucionario; la teoría original anterior sugiere que la hipertensión conduce a vasoconstricción cerebral autorreguladora, isquemia y posterior edema cerebral. $^{8}$

Sus manifestaciones clínicas van desde cefalea, alteración de la conciencia, alteraciones visuales y convulsiones, todo esto asociado con un perfil preeclámptico bioquímico sin alteraciones, incluso en ocasiones sin relación con trastornos hipertensivos del embarazo. ${ }^{9}$ En cuanto a su distribución anatómica, las anomalías se observan en la materia blanca cortical y subcortical del lóbulo occipital (circulación cerebral posterior). ${ }^{10}$

La resonancia magnética es el estudio de elección para esta entidad clínica. Los hallazgos típicos son el edema simétrico de la materia blanca en los hemisferios cerebrales posteriores, en particular, las regiones parieto-occipitales, pero existen variaciones. ${ }^{11}$ Se puede observar edema vasogénico en FLAIR y T2 con una hipointensidad, en las regiones periféricas, principalmente. ${ }^{12}$

En México, el estudio más amplio que se ha llevado a cabo hasta el momento, fue realizado por Morales y colaboradores, en el que se documentaron 176 pa- 
cientes positivas para PRES, demostrándose una relación directa de desarrollo de la patología hasta dos veces más con proteinuria mayor de $300 \mathrm{mg} / 24$ horas y estableciendo un alto riesgo de la enfermedad en pacientes con eclampsia anteparto, primigestas y menores de 21 años con una presión arterial media mayor de $120 \mathrm{mmHg}$ y más de dos crisis convulsivas. ${ }^{13}$ En nuestro estudio, la presentación clínica fue distinta a lo reportado en la bibliografía, ya que no se presentó cefalea ni déficit neurológico, con cifras tensionales normales, solamente manifestándose una convulsión, sin embargo, radiológicamente la topografía afectada fue en lóbulo parietal y occipital, con remisión total de las lesiones.

\section{CONCLUSIÓN}

EI PRES es una entidad extremadamente rara de prevalencia desconocida. El caso clínico descrito cursó con una evolución gestacional de características normales y sin alteraciones registradas durante su embarazo. La presentación anteparto de las crisis convulsivas orientó al diagnóstico de eclampsia, sin embargo, la ausencia de antecedentes de crisis convulsivas y encontrar una tomografía de cráneo simple normal obligan a diferenciarlo de síndrome de PRES. Radiológicamente se muestra como hiperintensidad en resonancia magnética, con predominio en lóbulo parietal y occipital. Los diagnósticos diferenciales han llevado a un aumento en la incidencia de este tipo de patologías. El pronóstico es favorable cuando el diagnóstico y el abordaje son oportunos, logrando recuperación ad integrum.

\section{AGRADECIMIENTOS}

A las instalaciones del Instituto Mexicano del Seguro Social y al personal de Unidad de Cuidados Intensivos Metabólicos.

\section{BIBLIOGRAFÍA}

1. Hinchey J, Chaves C, Appignani B, Breen J, Pao L, Wang A, et al. Un síndrome de leucoencefalopatía reversible posterior. $N$ Engl J Med. 1996;334:494-500.
2. Fischer M, Schmutzhard E. Síndrome de encefalopatía posterior reversible. J Neurol [En línea]. 2017 [consultado el 9 de diciembre de 2019];264:1608-1616. Disponible en: https://www.ncbi.nlm.nih. gov/pubmed/28054130. doi: 10.1007/s00415-016-8377-8.

3. Okamoto K, Motohashi K, Fujiwara H, Ishihara T, Ninomiya I, Onodera O, et al. PRES: síndrome de encefalopatía reversible posterior. Brain Nerve [En línea]. 2017 [consultado el 12 de diciembre de 2019];69(2):129-141. doi: 10.11477/ mf.1416200653.

4. Domínguez-Fuentes B, García-Gil D. Leucoencefalopatía posterior reversible en una paciente con eclampsia posparto. Med Intensiva. 2008;32(7):361-363.

5. Covarrubias DJ, Luetmer PH, Campeau NG. Síndrome de encefalopatía reversible posterior: utilidad pronóstica de las imágenes de RM ponderadas por difusión cuantitativa. AJNR Am J Neuroradiol. 2002;23:1038-48.

6. Luzaino G. Cloft H, Hemstreet MK, et al. Reversible posterior leukoencephalopaty following organ transplantation. Description of two cases. Clin Neurol Neurosurg. 1997;99:222.

7. Strandgaard S, Paulson OB. Autorregulación cerebral. Stroke. 1984;15:413.

8. Lamy C, Oppenheim C, Mas JL. Posterior reversible encephalopathy syndrome. Handb Clin Neurol. 2014;121:1687-1701.

9. Fugate JE1, Claassen DO, Cloft HJ, Kallmes DF, Kozak OS, Rabinstein A. Síndrome de encefalopatía reversible posterior: hallazgos clínicos y radiológicos asociados. Mayo Clin Proc. 2010;85:427-32.

10. Kalimo H, Fredriksson K, Nordborg C, Auer RN, Olsson Y, Johansson B. La propagación del edema cerebral en la lesión cerebral hipertensiva. Med Biol. 1986;64:133-7.

11. McKinney AM, Jagadeesan BD, Truwit CL. Síndrome de encefalopatía reversible posterior de variante central: afectación del tronco encefálico o de los ganglios basales sin edema cerebral cortical o subcortical. AJR Am J Roentgenol. 2013;201:631.

12. Staykov D, Schwab S. Síndrome de encefalopatía reversible posterior. J Intensive Care Med. 2012;27:11-24.

13. Morales-Muñoz G, Mercedes-Acuña A, Padilla-Chávez R. Síndrome de encefalopatía posterior reversible en eclampsia en el Hospital Regional de Alta Especialidad de la Mujer de Tabasco, México. Med Crit [En línea]. 2017 [consultado el 13 de diciembre de 2019];31(2):60-64.

Financiamiento: Autofinanciado por los autores.

Conflicto de intereses: Los autores declaran no tener conflicto de intereses.

\section{Correspondencia:}

Dra. Cibeles Arrambí Díaz

Departamento de Urgencias del Hospital Regional No. 1 del Instituto Mexicano del Seguro Social.

Calle Puente Alcántara Núm. 910,

Ciudad Obregón, Sonora.

Tel: (644) 1392667

E-mail: cibelesarrambi@hotmail.com 